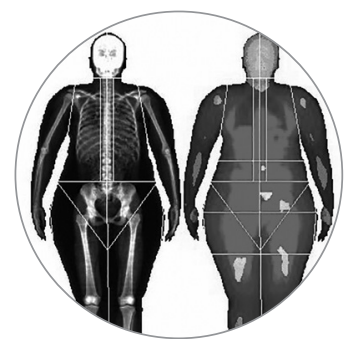

\title{
Cambios en la densidad mineral ósea y en la composición corporal en pacientes obesos sometidos a cirugía bariátrica
}

\section{Changes in Bone Mineral Density and Body Composition in Obese Patients Undergoing Bariatric Surgery}

\author{
Sara Jaimes Niño \\ Sergio Valencia ${ }^{2}$ \\ Gustavo Triana ${ }^{3}$ \\ Javier Andrés Romero
}

\begin{tabular}{|l|}
\hline 2 \\
\hline Palabras clave (DeCS) \\
\hline $\begin{array}{l}\text { Composición corporal } \\
\text { Densitometría } \\
\text { Cirugía bariátrica } \\
\text { Obesidad } \\
\text { Sarcopenia }\end{array}$ \\
\hline Key words (MeSH) \\
\hline Body composition \\
Densitometry \\
Bariatric surgery \\
Obesity \\
Sarcopenia
\end{tabular}

Médica cirujana, Pontificia Universidad Javeriana. Residente de Radiología, Universidad El Bosque. Bogotá, Colombia.

2 Médico general, Universidad de los Andes. Magíster en Epidemiología, Universidad El Bosque. Bogotá, Colombia.

${ }^{3}$ Radiólogo y Magister en Epidemiología, Pontificia Universidad Javeriana. Bogotá, Colombia.

${ }^{4}$ Departamento de Imágenes Diagnósticas, Hospital Universitario Fundación Santa Fe de Bogotá. Bogotá, Colombia.

\section{Resumen}

La obesidad es un problema de salud pública que afecta de forma significativa a la población mundial, aumentando los índices de morbilidad y mortalidad. A pesar de que existen múltiples tratamientos para la obesidad, el más efectivo a largo plazo es el quirúrgico. Las cirugías bariátricas, como el bypass gástrico en forma de Y-de-Roux y la manga gástrica, han logrado no solamente rebajar la masa corporal a expensas de la reducción de la masa grasa, sino que han disminuido la prevalencia de comorbilidades como diabetes mellitus tipo 2, dislipidemia, hipertensión arterial, entre otras. Sin embargo, las cirugías bariátricas también han demostrado tener efectos negativos sobre el índice musculoesquelético y la densidad mineral ósea, variables que deben ser tenidas en cuenta durante la valoración prequirúrgica y las recomendaciones posquirúrgicas. A partir de los resultados del estudio se puede inferir que la cirugía bariátrica ocasiona osteopenia/osteoporosis y sarcopenia a largo plazo basados en la disminución estadísticamente significativa del contenido mineral óseo (CMO) y del tejido magro libre de grasa a corto plazo, por lo cual se propone un seguimiento a largo plazo (mayor de 12 meses) de dichos pacientes.

\section{Summary}

Obesity is a public health problem that significantly affects the world population, increasing morbidity and mortality rates. Although there are multiple treatments for obesity, the most effective treatment in the long term is surgical. Bariatric surgeries, such as Y-de-Roux gastric bypass and gastric sleeve, have not only reduced body mass at the expense of reducing fat mass, but have also reduced the prevalence of comorbidities such as type 2 diabetes mellitus, dyslipidemia, arterial hypertension, among others. However, bariatric surgeries have also been shown to have negative effects on the musculoskeletal index and bone mineral density, variables that must be taken into account during pre-surgical assessment and post-surgical recommendations. From the results of the study, it can be inferred that bariatric surgery causes osteopenia / osteoporosis and sarcopenia in the long term based on the statistically significant decrease in bone mineral content (BMC) and fat-free lean tissue in the short term, which is why a long-term follow-up (longer than 12 months) of these patients is proposed.

\section{Introducción}

La obesidad es un problema de salud pública que afecta de forma significativa a la población mundial, con altos índices de morbilidad y mortalidad. Según la Organización Mundial de la Salud (OMS), la obesidad se ha triplicado en el mundo desde 1975. En 2016 el $39 \%$ de la población adulta (personas mayores de18 años) presentaba sobrepeso, mientras que $13 \%$ de esta población era considerada obesa (1). Se han desarrollado múltiples tratamientos, de los cuales, las cirugías bariátricas son las de mayor índice de efectividad para la reducción de peso y de comorbilidades a largo plazo. Sin embargo, estos procedimientos quirúrgicos han mostrado efectos secundarios, como osteopenia y sarcopenia, hallazgos parcialmente entendidos y que no se han estudiado a profundidad.

La introducción del estudio de la composición corporal y de la osteodensitometría han permitido analizar dichos cambios secundarios a las cirugías bariátricas, evaluar el comportamiento de los diferentes componentes corporales - previo y posterior al procedimiento quirúrgico-y la relación entre estos.

En nuestra institución se realizan múltiples cirugías bariátricas con un estricto seguimiento clínico y radiológico previo y posterior al procedimiento. Con base en los datos obtenidos de estos pacientes, se evalúa el comportamiento de los diferentes componentes corporales para establecer los efectos esperados y secundarios de estos procedimientos. 


\section{Marco teórico}

La obesidad es una enfermedad multifactorial con componentes genéticos, nutricionales y metabólicos (2). Según la OMS, se clasifica según el índice de masa corporal (IMC) (body mass index) - desarrollado hace aproximadamente 200 años por Quetelet — para describir la relación aritmética entre la masa corporal y la altura del paciente $\left(\mathrm{IMC}=\mathrm{kg} / \mathrm{m}^{2}\right)(2)$. Sin embargo, hoy en día se conoce que el IMC es un estimado matemático impreciso del tejido adiposo, que ignora múltiples factores asociados, y es poco útil para la predicción del riesgo cardiovascular de los pacientes (2).

El tejido adiposo y el musculoesquelético son más que una reserva de energía, también producen adipoquinas y mioquinas, las cuales participan activamente en el metabolismo de los lípidos y la glucosa como proteínas bioactivas (3). Debido a la función endocrinológica del tejido adiposo, es necesario clasificar la obesidad según la composición corporal de los pacientes, principalmente según su porcentaje de grasa corporal (PGC) (percent body fat) ya que este se encuentra en estricta relación con el riesgo cardiovascular (3). Sin embargo, no solo el PGC es determinante para definir el riesgo cardiovascular asociado a la obesidad; la distribución de la grasa en el cuerpo también es importante $(3,4)$.

La grasa visceral es aquella que se encuentra alrededor de los órganos intraabdominales y su incremento es secundario a la hipertrofia de las células adiposas ya existentes, asociado a vascularización inadecuada que conlleva fibrosis y secreción de citoquinas proinflamatorias (IL-1, IL-6, FNT $\alpha$ ); este tipo de grasa se relaciona con el tejido adiposo ectópico. Por otro lado, la grasa subcutánea es la que se encuentra por debajo de la piel, su aumento es secundario a la hiperplasia de células preadipocíticas asociado a una adecuada vascularización que conlleva expansión del tejido adiposo con mínima cantidad de fibrosis y mínima inflamación. Es de vital importancia diferenciar estos dos tipos de grasa debido a que es la grasa visceral la que constituye un factor de riesgo para el desarrollo de la dislipidemia y resistencia a la insulina, por lo tanto, es ante la cual se debe estar alerta. De acuerdo con esto, la distribución androide de la grasa (central) aumenta el riesgo cardiovascular en comparación con la distribución ginecoide de la grasa (periférica), lo cual también explica las anormalidades metabólicas en pacientes con peso normal $(4,5)$.

Como se mencionó, el músculo también es un órgano endocrino que se afecta por exceso de tejido adiposo, y produce sarcopenia — pérdida de masa muscular, con posterior debilidad y limitación para los movimientos- El concepto de obesidad sarcopénica fue desarrollado inicialmente por Roubenoff, quien descubrió que el aumento de citoquinas inflamatorias producidas por el exceso de tejido adiposo, especialmente la grasa visceral, acelera el catabolismo muscular a través de dos vías: directa —alterando el balance aminoácido del músculo — e indirecta — disminuyendo la sensibilidad a la insulina - lo que lleva a la sarcopenia $(2,6)$.

La obesidad sarcopénica es un problema de salud pública asociado a limitaciones funcionales y aumento de la mortalidad, su incidencia puede reducirse en aproximadamente $20 \%$ con un diagnóstico temprano y adecuado tratamiento (1). De acuerdo con esto, el dictamen de obesidad se debe realizar utilizando varios métodos diagnósticos, incluyendo la composición corporal. La prevención y tratamiento de la obesidad debe incluir cambios en el estilo de vida (dieta apropiada, aumento del ejercicio), terapia comportamental y farmacoterapia; sin embargo, estas medidas no son suficientes para algunos pacientes, principalmente aquellos pacientes cuyo IMC $>40 \mathrm{~kg} / \mathrm{m}^{2}$. En estos pacientes se debe considerar adicionalmente un tratamiento quirúrgico, el cual es muy efectivo en la reducción de peso a largo plazo y en la diminución de las comorbilidades y de la mortalidad $(7,8)$.

La cirugía bariátrica se recomienda para pacientes con $\mathrm{IMC}>35$ $\mathrm{kg} / \mathrm{m}^{2}$ (principalmente pacientes con IMC $>40 \mathrm{~kg} / \mathrm{m}^{2}$ ) quienes tienen comorbilidades asociadas, como síndrome metabólico y diabetes mellitus tipo 2 (2). Hay varios tipos de cirugía bariátrica: bypass gástrico en Y-de-Roux (BGYR), manga gástrica, banda gástrica e interruptor duodenal. Para este trabajo se incluyeron los pacientes sometidos a las dos primeras técnicas $(2,9)$.

Los cambios en el tracto gastrointestinal secundarios principalmente al BGYR son en la morfología de la mucosa intestinal y en su función metabólica. La hipertrofia intestinal no solo es un cambio morfológico posquirúrgico, sino que constituye una respuesta fisiológica del cuerpo para evitar la malabsorción; sin embargo, es de esperarse que la absorción intestinal posquirúrgica no sea igual a la absorción intestinal prequirúrgica, secundario a la deficiencia en la absorción calórica y de grasas. Debido a esto, las cirugías bariátricas también son llamadas cirugías metabólicas $(10,11)$.

Durante la última década se ha generado una gran preocupación en torno a la cirugía bariátrica, ya que esta no solo disminuye la masa corporal, sino que produce cambios negativos en la densidad mineral ósea (5). El calcio, el mineral más abundante del organismo humano, se absorbe principalmente en la primera porción del intestino delgado (duodeno), el cual, después del BGYR se encuentra aislado del tracto gastrointestinal "activo" o alimenticio (tracto gastrointestinal que se encuentra en contacto con los alimentos); como consecuencia, hay una marcada disminución de la mineralización ósea que afecta negativamente la estabilidad del hueso (12). La disminución en la densidad mineral ósea persiste después del primer año posquirúrgico, debido a la malabsorción de calcio y de vitamina D $(13,14)$. Adicionalmente se disminuye la grasa magra corporal. No obstante, la reducción del peso corporal es principalmente a expensas de la disminución de la masa grasa $(13,15)$.

Las cirugías bariátricas han demostrado cumplir adecuadamente con sus objetivos principales: disminución de la masa corporal, principalmente a expensas de componente graso, y descenso en la incidencia de comorbilidades asociadas a la obesidad (hipertensión arterial, diabetes mellitus tipo 2, dislipidemia, entre otros) (13). Sin embargo, han surgido efectos no esperados del procedimiento que deben tenerse en cuenta para la evaluación de los pacientes previo a la cirugía y las recomendaciones posquirúrgicas: uno de estos eventos es la disminución de la densidad mineral ósea. Por esto, actualmente a los pacientes se les administra suplemento de calcio (600-1200 mg/día) y de vitamina D (400-800 UI/día) para evitar este efecto secundario y el aumento en el riesgo de fracturas $(13,14)$.

Retomando lo discutido al inicio, las medidas antropométricas, como la masa corporal (peso), altura y circunferencia abdominal, permiten obtener una moderada apreciación del grado de obesidad (según IMC) y el riesgo cardiovascular de los pacientes. No obstante, la composición corporal que se obtiene mediante absorciometría por rayos-X de energía dual (DEXA, del inglés Dual-energy X-ray absorptiometry), permite observar de forma más precisa y en menor 
tiempo esta información, pues muestra la masa corporal total, el índice de masa corporal (IMC), el tejido adiposo visceral estimado (TAV), el tejido magro libre de grasa, la relación androide/ginecoide (A/G), la tasa metabólica en reposo, la densidad mineral ósea (DMO) y el contenido mineral óseo (CMO) $(13,16)$. Adicionalmente, la composición corporal suministra información parcial acerca de la masa muscular representada en el índice musculoesquelético relativo (índice MSK), el cual permite definir el estado de sarcopenia de los pacientes, así como estimar la masa muscular apendicular (masa magra en extremidades) ajustada al IMC del paciente; se excluye del análisis la masa muscular troncal por su dificultad para la medición. Los valores de normalidad establecidos para el índice musculoesquelético relativo son $<0,789$ en hombres y $<0,512$ en mujeres $(6,15,17)$.

Algunas de las limitaciones de este método diagnóstico son su incapacidad de comparar TAV versus tejido adiposo subcutáneo estimado (TAS), el sobreestimar los valores obtenidos y su incompetencia para valorar el contenido de agua del cuerpo, asumiendo que los tejidos blandos se encuentran hidratados (18). Sin embargo, la alta disponibilidad y bajo costo en comparación con otros métodos diagnósticos de similares características (tomografía computarizada [TC] y resonancia magnética $[\mathrm{RM}]$ ), hacen del DEXA el método de elección (18).

La osteodensitometría utiliza la misma tecnología de DEXA para obtener información específica acerca de la DMO y CMO en la columna vertebral, cadera y cuello femoral; de esta forma puede predecir el riesgo de fractura del paciente (pacientes entre 40 a 90 años), utilizando algoritmos matemáticos específicos como el FRAX (19).

\section{Métodos}

Se realizó un estudio retrospectivo y descriptivo, con pacientes que se sometieron a cirugía bariátrica en el Hospital Universitario Fundación Santa Fe de Bogotá (HU-FSFB) entre 1 de enero de 2015 y el 31 de mayo de 2017. Como criterios de inclusión, los pacientes debían ser mayores de 18 años, tener un índice de masa corporal mayor a 35 y densitometría pre y posquirúrgica; se excluyeron del estudio los pacientes con antecedente de cirugía bariátrica previa que iban a ser sometidos a una reintervención, debido a que, al tener una cirugía anterior, su estado metabólico al inicio del estudio se encuentra alterado.

Después de la selección de pacientes se revisaron las historias clínicas y las imágenes diagnósticas, con el fin de recopilar sus datos sociodemográficos y clínicos de acuerdo con el formato de recolección de datos.

\section{Análisis estadístico}

El análisis estadístico se ejecutó en el programa Stata $13{ }^{\circledR}$ (StataCorp), univariado, para establecer las frecuencias relativas y las proporciones de las variables categóricas. Adicionalmente, se analizaron las variables continuas para obtener medidas de tendencia central (media, mediana) y de dispersión (rango intercuartil, desviación estándar).

Finalmente, para evaluar el efecto de la cirugía en variables como la composición corporal y la densidad mineral ósea, la prueba t de Student de datos pareados o la prueba de rango con signo de Wilcoxon, según normalidad. El coeficiente de correlación de Spearman se utilizó para evaluar la relación lineal entre los cambios en la composición corporal y la densidad mineral ósea, así como en las variables clínicas y demográficas. De igual forma, se realizó un modelo de regresión para evaluar de forma multivariada la asociación entre la reducción de la densidad mineral ósea, la composición corporal y las variables clínicas y demográficas

\section{Resultados}

Se revisaron 152 historias clínicas de pacientes sometidos a cirugía bariátrica entre el 1 de enero de 2015 y el 31 de mayo de 2017. Se analizaron las características demográficas y los resultados clínicos de 22 pacientes, 130 pacientes se excluyeron dado que no presentaban las medidas previas y 6-12 meses posteriores al procedimiento. De los 22 pacientes analizados, el 80,8 \% fueron mujeres, el promedio de edad fue 43,61 años, con una desviación estándar de 10,49 años.

Durante el análisis se encontró una disminución estadísticamente significativa del índice de masa corporal (IMC) 32,69 vs. 24,13 ( $p=0,001$ ), tejido adiposo visceral estimado (VAT) $1174,26 \mathrm{~g}$ vs. 486,27 g $(p>0,001)$, contenido mineral óseo (CMO) 2563,78 g vs. 2476,62 g (p >0,001), tejido magro libre de grasa $44,85 \mathrm{~g}$ vs. $40,68 \mathrm{~g}$ ( $\mathrm{p}>0,001)$, tejido graso $46,08 \%$ vs. $34,07 \%(p>0,001)$ y la relación androide/ginecoide 1,03 vs. $0,87(p>0,001)$. A pesar de que la densidad mineral ósea - (DMO) $1,24 \mathrm{~g} / \mathrm{cm}^{2}$ vs. $1,27 \mathrm{~g} / \mathrm{cm}^{2}(p 0,014)$ - y el índice musculoesquelético relativo $-7,89 \mathrm{~kg} / \mathrm{m}^{2}$ vs. $6,53 \mathrm{~kg} / \mathrm{m}^{2}(p 0,68)$ - presentaron una disminución estadísticamente no significativa, consideramos que este tipo de disminución se debe al corto periodo de seguimiento de los pacientes; ya que la disminución estadísticamente significativa del CMO y del tejido magro libre de grasa predicen una probable disminución estadísticamente significativa de la DMO y el índice musculoesquelético a largo plazo, lo cual requeriría un periodo de seguimiento mayor a 12 meses (tabla 1).

\section{Tabla 1. Registro del cambio en las variables de la composición corporal}

\begin{tabular}{|c|c|c|c|c|c|}
\hline Composición & \multicolumn{2}{|c|}{ Antes } & \multicolumn{2}{|c|}{ Después } & \\
\hline Variables & Media & $\begin{array}{c}\text { Desviación } \\
\text { estándar }\end{array}$ & Media & $\begin{array}{c}\text { Desviación } \\
\text { estándar }\end{array}$ & $\begin{array}{l}\text { Valor } \\
\text { de } p\end{array}$ \\
\hline IMC & 32,69 & $(2,68)$ & 24,13 & $(2,84)$ & 0,001 \\
\hline $\mathrm{CMO}(\mathrm{g})$ & 2563,78 & $(356,92)$ & 2476,62 & $(349,16)$ & $\stackrel{>}{0,001}$ \\
\hline $\begin{array}{c}\text { DMO } \\
\left(\mathrm{g} / \mathrm{cm}^{2}\right)\end{array}$ & 1,24 & $(0,09)$ & 1,27 & $(0,36)$ & $0,014^{*}$ \\
\hline $\mathrm{TM}(\mathrm{g})$ & 44,85 & $(10,08)$ & 40,68 & $(7,30)$ & $\stackrel{>}{>, 001^{*}}$ \\
\hline TG (\%) & 46,08 & $(5,41)$ & 34,07 & $(7,61)$ & $\stackrel{>}{0,001}$ \\
\hline TAV (g) & 1174,26 & $(857,20)$ & 486,27 & $(489,02)$ & $\stackrel{>}{>, 001^{*}}$ \\
\hline$a / g$ & 1,03 & $(0,17)$ & 0,87 & $(0,26)$ & $\begin{array}{c}> \\
0,001\end{array}$ \\
\hline $\begin{array}{c}\text { Índice MSK } \\
\left(\mathrm{kg} / \mathrm{m}^{2}\right)\end{array}$ & 7,89 & $(1,19)$ & 6,53 & $(0,86)$ & $0,68^{*}$ \\
\hline
\end{tabular}

$\mathrm{IMC}=$ índice de masa corporal. $\mathrm{CMO}=$ contenido mineral óseo. $\mathrm{DMO}=$ densidad mineral ósea. TM = tejido magro libre de grasa. TG = tejido graso TAV = tejido adiposo visceral estimado. $\mathrm{a} / \mathrm{g}$ = relación androide/ginecoide MSK: índice musculoesquelético.

*Distribución no normal, valor de p estimado con prueba de Wilcoxon. 
Posteriormente, se realizó el test de correlación de Spearman, el cual determina la correlación de asociación o interdependencia entre dos variables aleatorias, ya sean continuas o discretas. Durante esta prueba se estableció un coeficiente de correlación significativa entre múltiples variables de la composición corporal (tabla 2); la variable con mayor correlación fue el índice de masa corporal (IMC). Una de las relaciones entre variables que resulta importante enfatizar es el coeficiente de correlación significativo $(p=<0,00)$ entre el CMO y la DMO (figura 1), lo cual establece una fuerte relación de asociación (figuras 2 y 3 ).

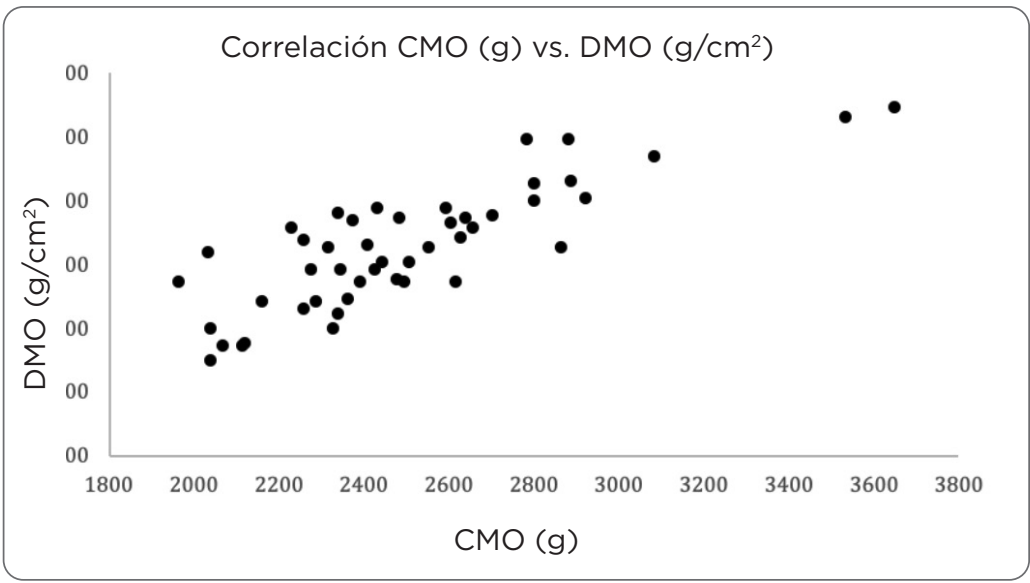

Figura 1. Se observa la correlación entre el CMO y la DMO demostrando que al aumentar/disminuir el CMO, la DMO presentará el mismo comportamiento.

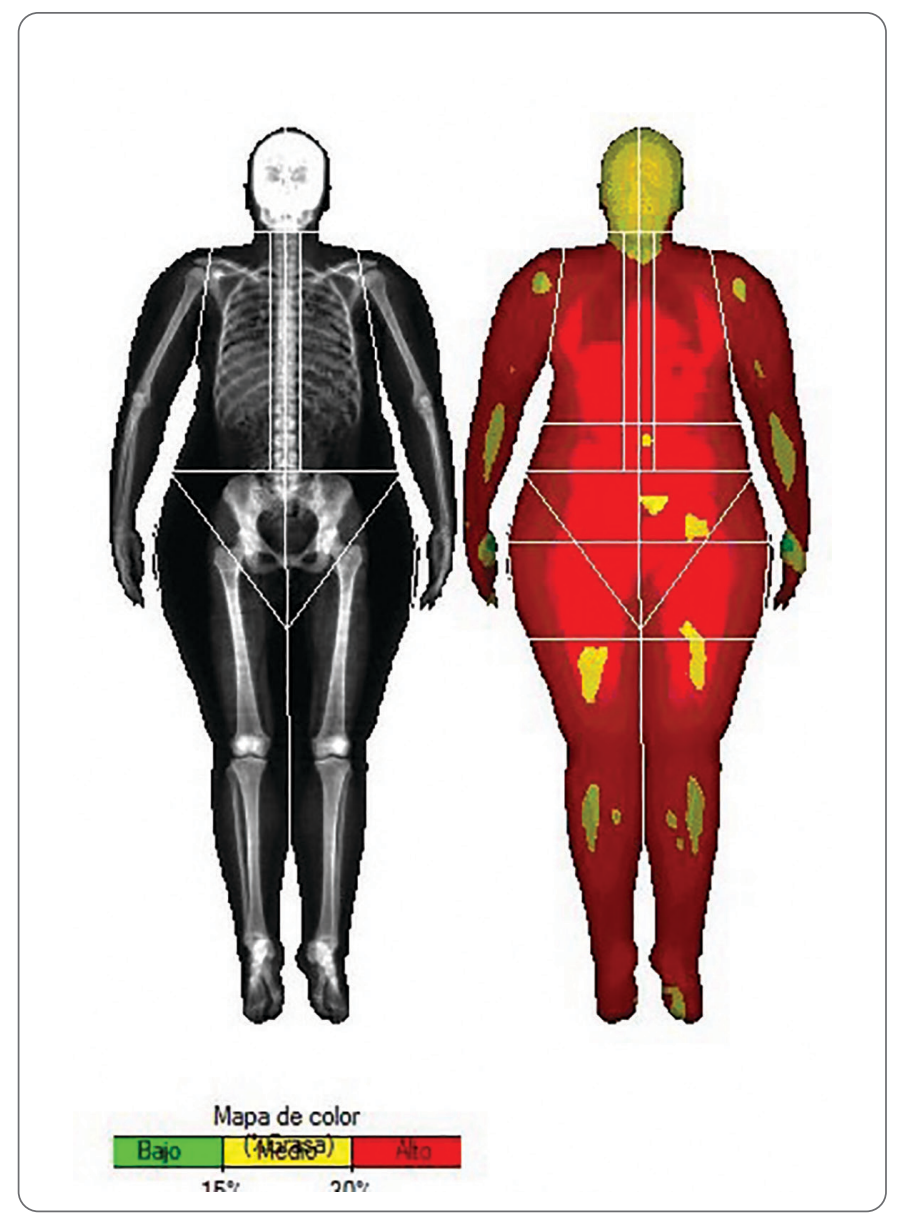

Figura 2. Estudio de composición corporal previo a procedimiento quirúrgico. Mujer de 30,1 años de edad con altura de $161 \mathrm{~cm}$, previo a intervención quirúrgica con masa corporal total de $93,5 \mathrm{~kg}$, tejido adiposo visceral estimado de $526 \mathrm{~g}$, índice musculoesquelético relativo de 8,02 $\mathrm{kg} / \mathrm{m}^{2}$, tejido graso $52 \%$ y densidad mineral ósea de $1,609 \mathrm{~g} / \mathrm{cm}^{2}$.

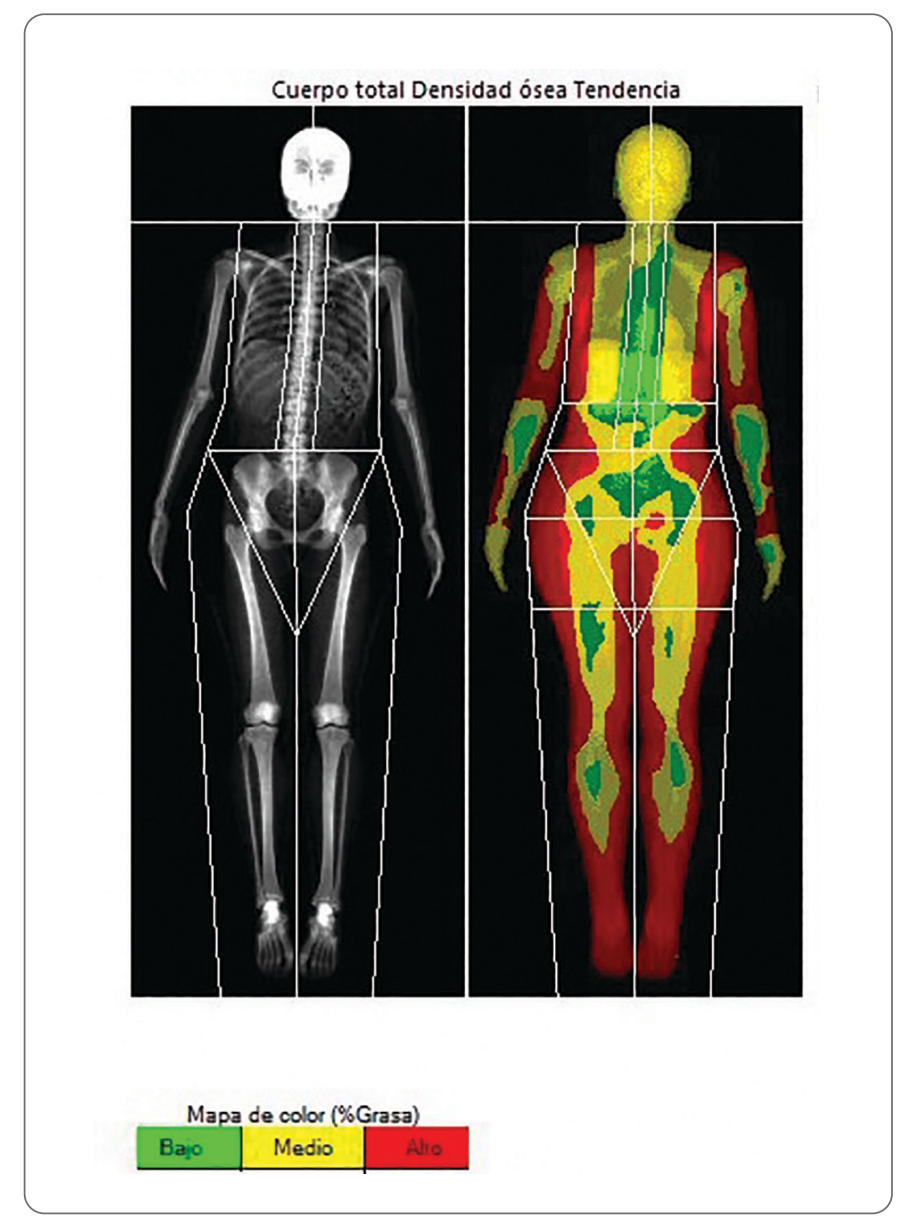

Figura 3. Estudio de composición corporal posterior a procedimiento quirúrgico. Mujer de 31,5 años de edad con altura de $161 \mathrm{~cm}$, posterior a intervención quirúrgica con masa corporal total de $58,3 \mathrm{~kg}$, tejido adiposo visceral estimado de $61 \mathrm{~g}$, índice musculoesquelético relativo de $6,23 \mathrm{~kg} / \mathrm{m}^{2}$, tejido graso $30,5 \%$ y densidad mineral ósea de $1,471 \mathrm{~g} / \mathrm{cm}^{2}$. 
Tabla 2. Correlación de Spearman entre variables

\begin{tabular}{|c|c|c|c|c|c|c|c|c|c|}
\hline & & CMO & DMO & IMC & TM & TG & VAT & $a / g$ & Índice MSK \\
\hline \multirow{2}{*}{ CMO } & $\begin{array}{l}\text { Coeficiente de } \\
\text { correlación }\end{array}$ & 1,000 & $0,788^{* *}$ & 0,150 & $0,807^{* *}$ & $-0,172$ & 0,207 & 0,077 & $0,464^{* *}$ \\
\hline & Significancia (bilateral) & & 0,000 & 0,315 & 0,000 & 0,237 & 0,153 & 0,601 & 0,001 \\
\hline \multirow{2}{*}{ DMO } & $\begin{array}{l}\text { Coeficiente de } \\
\text { correlación }\end{array}$ & $0,788^{* *}$ & 1,000 & $0,350^{*}$ & $0,491^{* *}$ & $-0,012$ & 0,219 & 0,175 & $0,435^{* *}$ \\
\hline & Significancia (bilateral) & 0,000 & & 0,015 & 0,000 & 0,936 & 0,127 & 0,225 & 0,002 \\
\hline \multirow{2}{*}{ IMC } & $\begin{array}{l}\text { Coeficiente de } \\
\text { correlación }\end{array}$ & 0,150 & $0,350^{*}$ & 1,000 & $0,426^{* *}$ & $0,743^{* *}$ & $0,657^{* *}$ & $0,610^{* *}$ & $0,730^{* *}$ \\
\hline & Significancia (bilateral) & 0,315 & 0,015 & & 0,002 & 0,000 & 0,000 & 0,000 & 0,000 \\
\hline \multirow{2}{*}{ TM } & $\begin{array}{l}\text { Coeficiente de } \\
\text { correlación }\end{array}$ & $0,807^{* *}$ & $0,491^{* *}$ & $0,426^{* *}$ & 1,000 & 0,024 & $0,491^{* *}$ & $0,341^{*}$ & $0,685^{* *}$ \\
\hline & Significancia (bilateral) & 0,000 & 0,000 & 0,002 & & 0,865 & 0,000 & 0,014 & 0,000 \\
\hline \multirow{2}{*}{ TG } & $\begin{array}{l}\text { Coeficiente de } \\
\text { correlación }\end{array}$ & $-0,172$ & $-0,012$ & $0,743^{* *}$ & 0,024 & 1,000 & $0,439 * *$ & $0,307^{*}$ & 0,218 \\
\hline & Significancia (bilateral) & 0,237 & 0,936 & 0,000 & 0,865 & & 0,001 & 0,029 & 0,137 \\
\hline \multirow{2}{*}{ TAV } & $\begin{array}{l}\text { Coeficiente de } \\
\text { correlación }\end{array}$ & 0,207 & 0,219 & $0,657^{* *}$ & $0,491^{* *}$ & $0,439 * *$ & 1,000 & $0,731^{* *}$ & $0,487^{* *}$ \\
\hline & Significancia (bilateral) & 0,153 & 0,127 & 0,000 & 0,000 & 0,001 & & 0,000 & 0,000 \\
\hline \multirow{2}{*}{$a / g$} & $\begin{array}{l}\text { Coeficiente de } \\
\text { correlación }\end{array}$ & 0,077 & 0,175 & $0,610^{* *}$ & $0,341^{*}$ & $0,307^{*}$ & $0,731^{* *}$ & 1,000 & $0,511^{* *}$ \\
\hline & Significancia (bilateral) & 0,601 & 0,225 & 0,000 & 0,014 & 0,029 & 0,000 & & 0,000 \\
\hline \multirow{2}{*}{$\begin{array}{l}\text { Índice } \\
\text { MSK }\end{array}$} & $\begin{array}{l}\text { Coeficiente de } \\
\text { correlación }\end{array}$ & $0,464^{* *}$ & $0,435^{* *}$ & $0,730^{* *}$ & $0,685^{* *}$ & 0,218 & $0,487^{* *}$ & $0,511^{* *}$ & 1,000 \\
\hline & Significancia (bilateral) & 0,001 & 0,002 & 0,000 & 0,000 & 0,137 & 0,000 & 0,000 & \\
\hline
\end{tabular}

IMC = índice de masa corporal. CMO = contenido mineral óseo. DMO = densidad mineral ósea. TM = tejido magro libre de grasa. TG = tejido graso. TAV = tejido adiposo visceral estimado. a/g = relación androide/ginecoide. MSK: índice musculoesquelético.

*La correlación es significativa en el nivel 0,05 (bilateral).

**La correlación es significativa en el nivel 0,01 (bilateral).

\section{Discusión y conclusiones}

La cirugía bariátrica demostró una disminución estadísticamente significativa del índice de masa corporal (IMC) y del tejido adiposo visceral estimado (TAV), cumpliendo adecuadamente con su objetivo principal. Sin embargo, estas no fueron las únicas variables con disminución estadísticamente significativa; este tipo de cambio también se observó en el tejido magro libre de grasa y en el componente mineral óseo (CMO).

La disminución del IMC en relación con la diminución del tejido magro libre de grasa predice una disminución estadísticamente significativa del índice musculoesquelético a mediano/largo plazo $(>12$ meses), debido a que este índice representa la estimación de masa magra en extremidades ajustada al IMC del paciente. Al producirse una disminución estadísticamente significativa del índice musculoesquelético, la cirugía bariátrica se constituye en un factor predictivo de sarcopenia.

Adicionalmente, la disminución estadísticamente significativa del CMO predice una disminución del mismo tipo en la densidad mineral ósea (DMO) a mediano/largo plazo (> 12 meses), al mostrar una relación directamente proporcional, lo cual, constituye a la cirugía bariátrica también en un factor predictivo de osteoporosis.
Los cambios en la composición corporal secundarios a cirugías bariátricas representan un amplio campo de investigación, abierto a nuevas propuestas de análisis y ávido por integración de conocimiento. Se requiere crear y ejecutar protocolos de estudio donde se establezca con claridad qué exámenes deben realizarse antes de la intervención y su frecuencia en el periodo posquirúrgico, para llevar a cabo un adecuado seguimiento; teniendo en cuenta que la duración del seguimiento deberá ser mayor a 12 meses. Mediante estos protocolos se podrán establecer correlaciones claras entre las diferentes variables de la composición corporal, que permitan predecir los cambios antes del procedimiento. De esta forma, se podrán definir las recomendaciones sobre el valor mínimo de densidad mineral ósea, contenido mineral óseo e índice musculoesquelético prequirúrgicos para evitar sarcopenia y osteoporosis en los pacientes sometidos a BGYR o manga gástrica.

\section{Referencias}

1. World Health Organization. Fact Sheet N311: Obesity and Overweight [internet]. 2018 [citado 2019 jun. 1]. Disponible en: https://www.who.int/news-room/fact-sheets/detail/ obesity-and-overweight 
2. De Lorenzo A, Soldati L, Sarlo F, Calvani M, Di Lorenzo N, Di Renzo L. New obesity classification criteria as a tool for bariatric surgery indication. World J Gastroenterol. 2016;22(2):681-703

3. Hallgreen CE, Hall KD. Allometric relationship between changes of visceral fat and total fat mass. Int J Obes (Lond). 2008;32(5):845-52.

4. Pories WJ, Dohm LG, Mansfield CJ. Beyond the BMI: the search for better guidelines for bariatric surgery. Obesity (Silver Spring). 2010;18(5):865-71

5. Lutz TA, Bueter M. The physiology underlying Roux-en-Y gastric bypass: a status report. Am J Physiol Regul Integr Comp Physiol. 2014;307(11):R1275-91.

6. Boutin RD, Yao L, Canter RJ, Lenchik L. Sarcopenia: Current Concepts and Imaging Implications. AJR Am J Roentgenol. 2015;205(3):W255-W66.

7. García-Caballero M, Reyes-Ortiz A, García M, Martínez-Moreno JM, Toval-Mata JA. Super obese behave different from simple and morbid obese patients in the changes of body composition after tailored one anastomosis gastric bypass (BAGUA). Nutr Hosp. 2014;29(5):1013-9.

8. Adams TD, Davidson LE, Litwin SE, Kim J, Kolotkin RL, Nanjee MN, et al. Weight and metabolic outcomes 12 years after gastric bypass. N Engl J Med. 2017;377(12):1143-55.

9. Valezi AC, Marson AC, Merguizo RA, Costa FL. Roux-en-Y gastric bypass: limb length and weight loss. Arq Bras Cir Dig. 2014;27 Suppl 1:56-8.

10. Thivel D, Brakonieki K, Duche P, Morio B, Boirie Y, Laferrere B. Surgical weight loss: impact on energy expenditure. Obes Surg. 2013;23(2):255-66

11. Tamboli RA, Hossain HA, Marks PA, Eckhauser AW, Rathmacher JA, Phillips SE, et al. Body composition and energy metabolism following Roux-en-Y gastric bypass surgery. Obesity (Silver Spring). 2010;18(9):1718-24

12. Schafer AL, Li X, Schwartz AV, Tufts LS, Wheeler AL, Grunfeld C, et al. Changes in vertebral bone marrow fat and bone mass after gastric bypass surgery: A pilot study. Bone. 2015;74:140-5.

13. Carrasco F, Ruz M, Rojas P, Csendes A, Rebolledo A, Codoceo J, et al. Changes in bone mineral density, body composition and adiponectin levels in morbidly obese patients after bariatric surgery. Obes Surg. 2009;19(1):41-6.

14. Johnson JM, Maher JW, DeMaria EJ, Downs RW, Wolfe LG, Kellum JM. The longterm effects of gastric bypass on vitamin D metabolism. Ann Surg. 2006;243(5):701-4; discussion 4-5.

15. Ciangura C, Bouillot JL, Lloret-Linares C, Poitou C, Veyrie N, Basdevant A, et al. Dynamics of change in total and regional body composition after gastric bypass in obese patients. Obesity (Silver Spring). 2010;18(4):760-5

16. Bazzocchi A, Diano D. Dual-energy x-ray absorptiometry in obesity. CMAJ 2014;186(1):48

17. Levitt DG, Beckman LM, Mager JR, Valentine B, Sibley SD, Beckman TR, et al. Comparison of DXA and water measurements of body fat following gastric bypass surgery and a physiological model of body water, fat, and muscle composition. J Appl Physiol (1985). 2010;109(3):786-95.

18. Silver HJ, Welch EB, Avison MJ, Niswender KD. Imaging body composition in obesity and weight loss: challenges and opportunities. Diabetes Metab Syndr Obes. 2010;3:337-47.

19. Maghrabi AH, Wolski K, Abood B, Licata A, Pothier C, Bhatt DL, et al. Two-year outcomes on bone density and fracture incidence in patients with T2DM randomized to bariatric surgery versus intensive medical therapy. Obesity (Silver Spring). $2015 ; 23(12): 2344-8$

\section{Correspondencia}

Sara Jaimes Niño

Carrera 7 \# 111-64

sc.jaimesn@gmail.com

Recibido para evaluación: 1 de agosto de 2019

Aceptado para publicación: 15 de noviembre de 2019 